\title{
Language Differences as a Barrier to Quality and Safety in Health Care: The Joint Commission Perspective
}

\author{
Paul M. Schyve, MD \\ The Joint Commission, Oakbrook Terrace, IL, USA.
}

Effective communication with patients is critical to the safety and quality of care. Barriers to this communication include differences in language, cultural differences, and low health literacy. Evidence-based practices that reduce these barriers must be integrated into, rather than just added to, health care work processes.

KEY WORDS: language differences; cultural differences; low health literacy; health care; information management; accreditation.

$\mathrm{J}$ Gen Intern Med 22(Suppl 2):360-1

DOI: $10.1007 / \mathrm{s} 11606-007-0365-3$

(c) Society of General Internal Medicine 2007

$\mathrm{W}$ hen a patient sees a clinician member of a treatment team, the clinician uses the available knowledge base (derived from her education and training, the literature, experience, decision-support systems, and protocols) to decide what data to collect about the patient and how to collect them. The clinician and other members of the team collect these data through conversations with the patient and the patient's family, observation of the patient, a physical and mental examination, laboratory testing, and imaging. The treatment team then synthesizes these patient-specific data with the evidence-based information in the available knowledge base to create new information: the patient's diagnosis and prognosis and, in the dialog with the patient, a treatment plan. Finally, the team disseminates this newly created information to the patient, the patient's family, other members of the treatment team, other professional caregivers, pharmacists, insurance companies, and others. Dissemination occurs through oral communication (e.g., in conversations with the patient, patient's family, and health care professionals), through writing (e.g., in consent forms, instructions, educational materials for patients, and in notes and instructions for other professional caregivers and pharmacists), and through electronic transmission (e.g., in pharmacy orders, insurance claims, and computerized, patient-accessible personal health records).

This collection of data, transformation of data into information, storage of data and information, and dissemination of information are the key processes that comprise information management. Today, in health care, much of this information management is in the form of oral and written communications between team members, patients, and patients' families. The more the care is patient- and family-centered, the more frequent the communication with the patient and the patient's family to understand the patient's perspective and to involve the patient in the treatment team itself.
Because much of medical care is really information management, this communication between treatment team members and the patient and patient's family is a core component of health care-it is more than an adjunct or facilitator of health care. Collection of accurate and comprehensive patientspecific data that are the basis for proper diagnosis and prognosis; involving the patient in treatment planning; eliciting informed consent; providing explanations, instructions, and education to the patient and the patient's family; and counseling and consoling the patient and family requires effective communication between the clinician, the patient, and the patient's family. Effective communication is communication that is comprehended by both participants; it is usually bidirectional between participants, and enables both participants to clarify the intended message. In the absence of comprehension, effective communication does not occur; when effective communication is absent, the provision of health care ends-or proceeds only with errors, poor quality, and risks to patient safety.

When patients with limited English proficiency are treated by physicians and other health professionals who are proficient only in English, 3 factors converge to create a "triple threat" to effective communication. First, the language differences themselves are a barrier to effective communication. Unfortunately, this language barrier is often not immediately evident. Instances in which patients identified themselves as (reasonably) proficient in English, but were not, have been reported, and a Joint Commission study found physicians and hospital staff who believed themselves to be sufficiently proficient in another language, but were not. ${ }^{1}$ Both the patient and the clinician can underestimate the language barrier between them.

Second, cultural differences-which are often associated with language differences-are a barrier to effective communication. One's culture affects one's understanding of a word or sentence and even one's perception of the world. To learn a language is not the same as understanding a culture-even those who share a common native language may not share a common culture. And not everyone born in the same place and speaking the same language necessarily shares all the features of a common culture. Therefore, there is a risk of either underestimating the effect of cultural differences or of stereotyping individuals by their culture. Both will interfere with the effectiveness of communication.

Third, low health literacy is a barrier to effective communication. ${ }^{2}$ Low health literacy in patients may be associated with language and cultural barriers, but is also found in patients who are proficient in English and who share the common U.S. culture. This latter group may be especially at risk of having their low health literacy go unrecognized. When language or 
cultural barriers are identified, it often leads the clinician to explore whether the patient understands her oral or written communication. But when the patient speaks the same language and is of the same culture as the clinician, too often the clinician assumes that the patient-in the absence of questions-understands. Many clinicians have belatedly discovered that a patient is functionally illiterate: the patient cannot read or write (general literacy), let alone understand the clinician's medical jargon or complex instructions (health literacy).

In an increasingly multilingual, multicultural society, providing safe, high-quality health care requires overcoming these 3 barriers to effective communication with patients and their families. When the Joint Commission first developed standards that required an organization to address the language needs of patients, it was in the context (subsequently bolstered by federal regulations) of the patient's right to be fully informed about his or her care. Later, the need to communicate effectively was recognized as an element of the quality of patient care. Today, effective communication-which takes into account language, cultural differences, and health literacy-is seen as a prerequisite to safe health care. Communication problems are the most frequent root cause of serious adverse events reported to the Joint Commission's Sentinel Event Database, ${ }^{3}$ and a Joint Commission study found that when patients suffer adverse outcomes from medical errors, the outcomes are more serious in limited English proficiency patients than in English-speaking patients. ${ }^{4}$ Patient rights, quality of care, and patient safety each in itself is sufficient to justify a commitment to effective communication. Together they make effective communication in health care obligatoryit is a critical component of the health care itself.

Yet often those health care organizations that are struggling to implement practices to reduce language, culture, and health literacy barriers to communication do not know which practices are most effective. ${ }^{1}$ And even when effective practices are known, their implementation in a reliable, sustainable, and efficient manner is challenging. This JGIM Supplement begins to provide some evidence-based solutions to this challenge. But more is to be learned, and the solutions that have been identified often must be adapted to individual organizations.

"Adaptation" does not adequately represent the challenge of implementation. Too often, evidence-based practices are simply bolted onto the existing system, thereby adding expense, increasing complexity, and potentially compromising existing work processes. Instead, an evidence-based practice should be incorporated into a redesign of the work processes of the system or subsystem of which it is to be a part. For example, it is more effective to incorporate a language interpretation service (an evidenced-based practice) into redesigned work processes in the emergency department (a subsystem in a hospital), rather than to simply graft it onto the department's current system of operations and expect it to integrate itself smoothly and efficiently without interfering with other work processes.

A health care delivery organization, even a clinician's office, is a complex system. Among the characteristics of a complex system are self-adaptation and nonlinear effects. That is, if the inputs to a complex system are changed, such as the addition of an interpreter service, the system itself will self-adapt-it will change to accommodate the new input. However, the selfadaptation may not achieve the goals desired by either the treatment team or the patient, but may instead lead to outcomes that are neither expected nor desired by the treatment team and the patient. The treatment team, patient, and patient's family need to anticipate and be alert to the probability of these unintended consequences. And because changes in complex systems have nonlinear effects, even the smallest change in 1 part of the system can lead to a large change elsewhere in the system. This can be an advantage when it is used to leverage the effect of a small change; it can also be a disadvantage if the large change is unexpected and adverse.

As a physician's or other clinician's office, clinic, hospital, or other health care delivery setting focuses its attention on improving the safety and quality of patient care, these observations generate a set of principles:

- Providing safe and high-quality patient care is dependent upon effective communication between health care professionals, patients, and patients' families.

- Effective communication requires the recognition and amelioration of 3 key barriers: language differences, cultural differences, and low health literacy.

- There is a growing body of evidence-based practices that address these 3 barriers (and of evidence that certain practices are ineffective or unsafe).

- For the implementation of these practices to be effective, reliable, and sustainable, the practices should be incorporated into the redesign of the relevant work processes in the health care delivery site (e.g., physician's practice, hospital), not just bolted onto the current system.

- Changes in the site's systems and processes are likely to produce unintended consequences; a prospective identification of these potential consequences should be undertaken before implementation, and vigilance for these consequences should follow implementation.

As the Joint Commission focuses the spotlight of its standards and accreditation process on patient safety and quality of care, these principles will guide its approach to removing communication barriers related to language, cultural differences, and low health literacy, as well as communication barriers arising from physical factors such as hearing, speech, and vision, and from health care interventions such as intubation. But do not await Joint Commission actions-if the goal of providing safe, high-quality care is to be achieved, the obligation of health care professionals and organizations to address linguistic, cultural, and health literacy barriers to patient communication is immediate.

Corresponding Author: Paul M. Schyve, MD; The Joint Commission, One Renaissance Boulevard, Oakbrook Terrace, IL 60181, USA (e-mail: pschyve@jointcommission.org).

\section{REFERENCES}

1. Wilson-Stronks A, Galvez E. Hospitals, Language, and Culture: A Snapshot of the Nation. Oakbrook Terrace, IL: The Joint Commission; 2007.

2. The Joint Commission. 'What Did the Doctor Say?:' Improving Health Literacy to Protect Patient Safety. Oakbrook Terrace, IL: The Joint Commission; 2007.

3. The Joint Commission. Root causes of sentinel events. Available at: http://www.jointcommission.org/NR/rdonlyres/FA465646-5F5F-4543AC8F-E8AF6571E372/o/root_cause_se.jpg. Accessed August 27, 2007.

4. Divi C, Koss RG, Schmaltz SP, Loeb JM. Language proficiency and adverse events in U.S. hospitals: a pilot study. Int $J$ Qual Health Care. 2007; 19:60-67. 